\title{
Diagnostic and prognostic value of carcinoembryonic antigen in pancreatic cancer: a systematic review and meta-analysis
}

This article was published in the following Dove Press journal:

OncoTargets and Therapy

15 September 2017

Number of times this article has been viewed

\author{
Qingcai Meng ${ }^{1-3, *}$ \\ Si Shi ${ }^{1-3, *}$ \\ Chen Liang ${ }^{1-3, *}$ \\ Dingkong Liang ${ }^{1-3}$ \\ Wenyan $\mathrm{Xu}^{1-3}$ \\ Shunrong $\mathrm{Ji}^{1-3}$ \\ Bo Zhang ${ }^{1-3}$ \\ Quanxing $\mathrm{Ni}^{1-3}$ \\ Jin $X u^{1-3}$ \\ Xianjun $\mathrm{Yu}^{\mathrm{I}-3}$ \\ 'Department of Pancreatic Surgery, \\ Fudan University Shanghai Cancer \\ Center, ${ }^{2}$ Department of Oncology, \\ Shanghai Medical College, ${ }^{3}$ Pancreatic \\ Cancer Institute, Fudan University, \\ Shanghai, People's Republic of China \\ *These authors contributed equally \\ to this work
}

Background: Carcinoembryonic antigen (CEA) is one of the most widely used tumor markers and is increased in 30\%-60\% of patients with pancreatic cancer. Although carbohydrate antigen 19-9 (CA19-9) is the most important serum biomarker in pancreatic cancer, the diagnostic and prognostic value of CEA is gradually being recognized.

Materials and methods: The MEDLINE, EMBASE, and Web of Science databases were searched for related literature published until January 2017. Diagnostic accuracy variables were pooled using the Meta-Disc software. The pooled hazard ratios (HRs) for prognostic data were calculated and analyzed using Stata software.

Results: A total of 3,650 participants enrolled in 19 studies met our inclusion criteria. The pooled sensitivity, specificity, positive likelihood ratio, and negative likelihood ratio of a CEA-based panel were 0.45 (95\% confidence interval [CI], 0.41-0.50), 0.89 (95\% CI, 0.86-0.91), 5.39 (95\% CI, 3.16-9.18), and 0.55 (95\% CI, 0.41-0.72), respectively. The area under the curve (AUC, 0.90) and Q-value (0.84) of the CEA-based panel indicated a significantly higher diagnostic accuracy compared with CEA or CA19-9 alone. Moreover, there was also a significant association between high levels of CEA and worse overall survival (HR, 1.43; 95\% CI, 1.31-1.56).

Conclusion: Our meta-analysis indicated that elevated serum CEA level, as a vital supplementary to CA19-9, can play an important role in the clinical diagnosis of pancreatic cancer patients and predict poor prognosis.

Keywords: carcinoembryonic antigen, pancreatic cancer, diagnosis, prognosis, meta-analysis

\section{Introduction}

Pancreatic cancer is one of the most aggressive malignancies and the fourth leading cause of cancer-related death. ${ }^{1}$ Despite advances in diagnostic and therapeutic strategies in the past 2 decades, the outcome of pancreatic cancer remains disappointing, and the 5-year survival rate is approximately $6 \%{ }^{2,3}$ Most pancreatic cancer patients are diagnosed at advanced stages due to a lack of specific symptoms and appropriate markers. Failure to identify patients with a high risk of metastasis and recurrence has also resulted in an unsatisfactory prognosis of pancreatic cancer patients.

Carcinoembryonic antigen (CEA), a glycoprotein with a molecular weight of $180-200 \mathrm{kDa}$, was initially isolated from fetal colon and colon cancer tissue in $1965 .{ }^{4}$ CEA is increased not only in colorectal cancer but also in various other types of cancer, including breast cancer, ${ }^{5}$ lung cancer, ${ }^{6}$ and thyroid cancer. ${ }^{7}$ Moreover, the serum level of CEA is increased in 30\%-60\% of pancreatic cancer patients. ${ }^{8,9}$ Carbohydrate antigen $19-9$ (CA19-9), the only biomarker currently recommended for clinical use by the National
Correspondence: Xianjun Yu; jin Xu Department of Pancreatic and Hepatobiliary Surgery, Fudan University Shanghai Cancer Center, 270 Dong'An Road, Shanghai 200032, People's Republic of China Tel +86 2I 64175590 ext I307 Fax +86216403 I446

Email yuxianjun@fudanpci.org; xujin@fudanpci.org 
Comprehensive Cancer Network guidelines for pancreatic cancer, has several limitations that should be considered when interpreting serum levels in a clinical setting. CA19-9 may lack sufficient sensitivity and specificity for certain patients with specific metastases or jaundice. ${ }^{10}$ Furthermore, sialylated Lewis antigen-negative individuals, constituting approximately $5 \%-10 \%$ of the population, have little or no secretion of CA19-9. ${ }^{11,12}$ For this proportion of the population, serum CEA was proposed as a potential marker to improve the diagnosis of pancreatic cancer. Thus, elevated CEA levels have been established as an independent predictor of poor survival of pancreatic cancer patients. ${ }^{13}$ Preoperative serum panel results of $\mathrm{CEA}^{+} / \mathrm{CA} 125^{+} / \mathrm{CA} 19-9 \geq 1,000 \mathrm{U} / \mathrm{mL}$ helped to identify a subgroup of patients with poor outcomes following surgery. ${ }^{14}$

Although many recent studies have focused on the relationship between CEA and pancreatic cancer, the results are still unclear. Therefore, this review initiated a comprehensive analysis to clarify the precise value of CEA in the diagnosis and prognosis of pancreatic cancer.

\section{Materials and methods Search strategy}

The investigation was conducted by searching the electronic databases such as MEDLINE, EMBASE, and Web of Science for all relevant articles published up to January 2017, using the following terms: "carcinoembryonic antigen" or "CEA," "diagnosis" or "prognosis," and "pancreatic cancer/tumor/ adenocarcinoma." The searches were supplemented by studying reference lists of the retrieved articles as well as relevant review articles. Two researchers independently assessed the eligibility of the potential studies following the guidelines of Preferred Reporting Items for Systematic Reviews and Meta-Analyses (PRISMA) statement. ${ }^{15}$

\section{Inclusion and exclusion criteria}

The eligible studies were selected according to the following inclusion criteria: 1) studied pancreatic cancer based on histopathological confirmation; 2) at least one of the diagnostic or prognostic value of CEA detection in pancreatic cancer patients was reported or able to be calculated from published data; and 3) samples were obtained from the peripheral blood. Exclusion criteria included the following: 1) duplicated studies using the same population or overlapping database; 2) literature published as reviews, case reports, letters, editorials, and expert opinions; and 3) studies published in a non-English language.

\section{Quality assessment}

The methodological quality of diagnosis in this review was assessed using the QUADAS-2 (Quality Assessment of Studies of Diagnostic Accuracy included in Systematic Reviews). ${ }^{16}$ The criteria consist of four key domains, including 1) patient selection; 2) conduct of the index test; 3) reference standard; and 4) flow and timing. All the four domains address the risk of bias, whereas the first three domains also consider concerns regarding applicability. Assessment results are presented as "low risk," "high risk," and "unclear risk."

The Newcastle-Ottawa Scale (NOS) was used to evaluate the methodological quality of prognosis in this review. ${ }^{17}$ In addition, the specific quality assessment of prognostic studies was estimated according to the approach of Hayden et al. ${ }^{18}$ This scale is an eight-item instrument that assesses the quality of the selection, comparability, exposure, and outcomes for study participants.

\section{Data extraction}

Two reviewers independently extracted the data from the selected studies and decided on controversial issues through discussion. The following information from each article was extracted: name of the first author, year of publication, country of study, number of samples, origin of samples, detection method, time of sampling, cutoff criteria, accuracy of diagnostic value (the number of true positives [TPs]; false positives [FPs]; true negatives [TNs]; false negatives $[\mathrm{FNs}]$ ), and survival data (hazard ratio [HR]). If not available, data were extracted using the method described by Tierney et $\mathrm{al}^{19}$ and Parmar et al. ${ }^{20}$

\section{Statistical analysis}

Diagnostic variables with corresponding 95\% confidence intervals (CIs), such as sensitivity, specificity, positive likelihood ratio (PLR), negative likelihood ratio (NLR), diagnostic odds ratio (DOR), and the summary receiver operating characteristic curve (SROC), were calculated and analyzed using the statistical methods described in the protocol. ${ }^{21}$ Pooled HRs of serum CEA levels for overall survival (OS) were calculated. The $I^{2}$ value was used to assess the statistical heterogeneity among the studies. A fixed-effects model was used for $I^{2}<50 \%$, whereas a random-effects model was used for $I^{2}>50 \%{ }^{22}$ The latent publication bias was assessed by a funnel plot. Meta-Disc 1.4 (XI Cochrane Colloquium, Barcelona, Spain) and Stata Version 12.0 software (StataCorp LP, College Station, TX, USA) were 
used to conduct statistical analysis. All $p$-values were two sided, and statistical significance was set at $p<0.05$.

\section{Results}

\section{Literature screening and study characteristics}

The flowchart of article selection is shown in Figure 1. We initially retrieved 875 published articles from the databases such as EMBASE, MEDLINE, and Web of Science. A total of 67 studies remained after manual screening of titles, abstracts, and keywords and removal of duplicates or irrelevant studies to the current analysis. A total of 48 articles were further removed according to the inclusion criteria or exclusion criteria. Finally, 19 studies were considered eligible for inclusion in the meta-analysis.

Detailed information on the included studies is summarized in Table 1. A total of 3,650 participants enrolled in 19 studies were analyzed for the association between serum CEA level and pancreatic cancer, and 2,329 (63.8\%) were included in 11 studies with prognosis analyses, including HRs, or standardized incidence ratios with 95\% CI. All participants enrolled in these studies were from America, Finland, Germany, Greece, Korea, Japan, China, Australia, Italy, and Turkey. A total of 15 studies adopted the critical values of CEA $(5 \mathrm{ng} / \mathrm{mL})$ as the cutoff value, with $8.4 \mathrm{ng} / \mathrm{mL}$, $2.5 \mathrm{ng} / \mathrm{mL}, 12.5 \mathrm{ng} / \mathrm{mL}$, and $3.47 \mathrm{ng} / \mathrm{mL}$ used in the other studies. All pancreatic cancer patients were confirmed by pathological examination of resected specimens or by fineneedle aspiration. The results of diagnosis quality assessment using the QUADAS-2 analysis are shown in Figure S1. The results of quality assessment according to NOS are presented in Table S1, and all studies achieved a score over 5 .

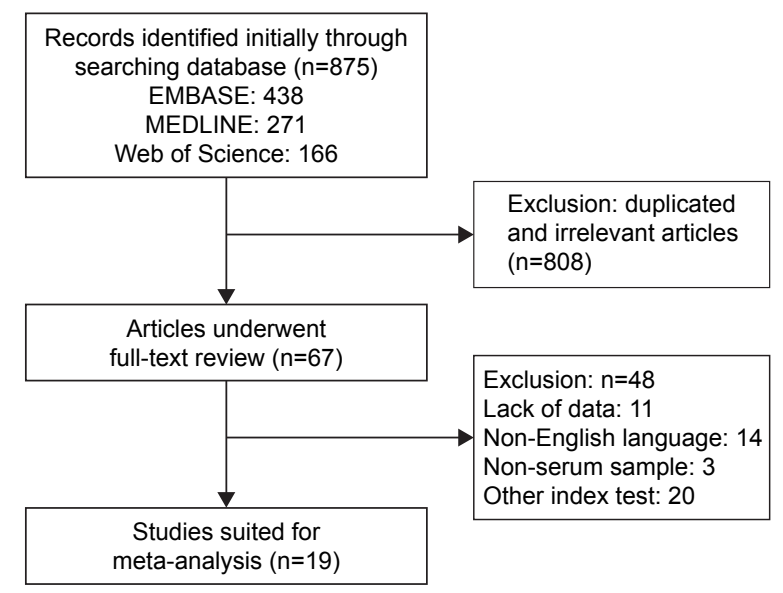

Figure I Literature review process.

\section{Meta-analysis results of diagnostic value}

Characteristics of the diagnostic analysis of the included studies are summarized in Table 2. Tumor markers, such as CA19-9, CEA, and the CEA-based tumor panel, were detected and evaluated on their diagnostic accuracy. Serum CEA alone had a pooled sensitivity of 0.43 (95\% CI, 0.39-0.47), specificity of 0.82 (95\% CI, 0.79-0.84), PLR of 2.40 (95\% CI, 1.68-3.43), and NLR of 0.71 (95\% CI, 0.65-0.78) (Figure S2). Similarly, the pooled accuracy of CA19-9 alone in this meta-analysis was also assessed (Figure S3). In addition, as shown in Figure 2, the overall accuracy of the CEA-based panel showed that the pooled sensitivity was 0.45 (95\% CI, 0.41-0.50), specificity was 0.89 (95\% CI, 0.86-0.91), PLR was 5.39 (95\% CI, 3.16-9.18), and NLR was 0.55 (95\% CI, 0.41-0.72). The DOR expressed how much greater the odds of having the disease are for individuals who have a positive test result compared with those who have a negative test result. The pooled DOR of CA19-9, CEA, and the CEA-based panel was 8.44 (95\% CI, 5.12-13.91), 3.57 (95\% CI, 2.29-5.57), and 19.20 (95\% CI, 6.45-57.18), respectively. $I^{2}$ values of the diagnostic variables were used to test the heterogeneity of these studies. The pooled indicators were calculated using the random-effects model due to the significant heterogeneity between these studies. The pooled results are summarized in Table 3. The SROC curve for the tumor diagnostic indicators, a comprehensive representative of test accuracy combining sensitivity with specificity, was drawn based on FP rates for the horizontal axis and TP rates for the vertical axis. An area under the curve (AUC) close to 1 reflects a well-performing diagnostic precision. ${ }^{23} \mathrm{We}$ also determined the Q-value, defined as the point of intersection of the SROC curve with a diagonal line extending from the left upper corner to the right lower corner. The $\mathrm{Q}$-value provides an overall measure of the discriminatory power of the diagnostic test. In this meta-analysis, the higher AUC (0.90) and Q-value (0.84) of the CEA-based panel are shown in Figure S4, compared with CA19-9 or CEA alone.

\section{Meta-analysis results of prognostic significance}

A total of 11 studies were available for calculating OS and pooled for survival analysis. As shown in Figure 3, the results indicated that a high level of CEA in pancreatic cancer was associated with worse OS (HR, 1.43; 95\% CI, 1.31-1.56). A fixed-effects model was applied during calculation due to the moderate but insignificant heterogeneity 
Table I Summary of the studies included in the meta-analysis

\begin{tabular}{|c|c|c|c|c|c|c|c|c|c|}
\hline Study & Region & $\begin{array}{l}\text { Number of } \\
\text { participants }\end{array}$ & Result & $\begin{array}{l}\text { Survival } \\
\text { analysis }\end{array}$ & HR (95\% CI) & $\begin{array}{l}\text { CEA cutoff } \\
\text { criteria } \\
(\mathrm{ng} / \mathrm{mL})\end{array}$ & $\begin{array}{l}\text { Detection } \\
\text { method }\end{array}$ & $\begin{array}{l}\text { Sample } \\
\text { source }\end{array}$ & $\begin{array}{l}\text { Sample } \\
\text { time }\end{array}$ \\
\hline Benini et a ${ }^{36}$ & America & 193 & - & - & - & 8.4 & Immunoassay & Serum & BS \\
\hline Carpelan-Holmstrom et $\mathrm{al}^{39}$ & Finland & 191 & - & - & - & 5 & Immunoassay & Serum & BS \\
\hline Haas et $\mathrm{al}^{40}$ & Germany & 34 & OS & $U$ & $2.24(1.18-4.25)$ & 5 & Immunoassay & Serum & BS \\
\hline Tsavaris et $\mathrm{al}^{45}$ & Greece & 215 & OS & M & $1.58(1.14-2.20)$ & 5 & NR & Serum & BS \\
\hline Lee et $\mathrm{al}^{42}$ & Korea & 187 & OS & M & $1.52(1.03-2.23)$ & 5 & Immunoassay & Serum & BS \\
\hline Haglund ${ }^{32}$ & Finland & 201 & - & - & - & 2.5 & Immunoassay & Serum & BS \\
\hline Sakamoto et a ${ }^{38}$ & Japan & 61 & - & - & - & 12.5 & Immunoassay & Serum & BS \\
\hline Kanda et $\mathrm{al}^{44}$ & Japan & 166 & OS & $U$ & $1.06(0.79-1.42)$ & 5 & NR & Serum & BS \\
\hline $\mathrm{Ni}$ et $\mathrm{a}^{33}$ & China & 205 & - & - & - & 5 & Immunoassay & Serum & BS \\
\hline Reitz et al ${ }^{43}$ & Australia & 393 & OS & M & $1.27(1.00-1.61)$ & 5 & NR & Serum & NR \\
\hline Imaoka et al' ${ }^{13}$ & Japan & 433 & OS & KM & $1.77(1.42-2.20)$ & 5 & NR & Serum & BS \\
\hline Del Favero et $\mathrm{al}^{34}$ & Italy & 139 & - & - & - & 3.47 & Immunoassay & Serum & BS \\
\hline Liao et $\mathrm{al}^{35}$ & China & 150 & - & - & - & 5 & Immunoassay & Serum & BS \\
\hline Duraker et $\mathrm{al}^{37}$ & Turkey & 181 & - & - & - & 5 & Immunoassay & Serum & BS \\
\hline Distler et $\mathrm{al}^{46}$ & Germany & 259 & OS & M & $1.299(1.127-1.496)$ & 5 & Immunoassay & Serum & BS \\
\hline Papadoniou et a ${ }^{47}$ & Greece & 215 & OS & $M$ & $1.58(1.14-2.20)$ & 5 & NR & Serum & BS \\
\hline Kim et $\mathrm{a}^{41}$ & Korea & 144 & OS & M & $2.60(1.22-5.55)$ & 5 & NR & Serum & AS \\
\hline Gu et $\mathrm{al}^{31}$ & China & 132 & OS & KM & $1.023(0.975-3.208)$ & 5 & Immunoassay & Serum & BS \\
\hline Xu et $\mathrm{al}^{48}$ & China & 151 & OS & M & $2.654(1.643-4.289)$ & 5 & Immunoassay & Serum & AS \\
\hline
\end{tabular}

Note: -, indicates not mentioned.

Abbreviations: AS, after treatment; BS, before treatment; CEA, carcinoembryonic antigen; HR, hazard ratio; KM, Kaplan-Meier analysis; M, multivariate analysis; NR, not reported; OS, overall survival; $U$, univariate analysis.

$\left(I^{2}=56.6 \%, p=0.011\right)$. In the subgroup analyses by ethnicity, six studies were conducted in Asian population (two in China, two in Japan, and two in Korea) and the remaining five in Caucasian population (one in Australia, two in Greece, and two in Germany). Regardless of whether the cases were from Asian or Caucasian populations, a high level of CEA was still a significant predictor of poor OS of pancreatic cancer (Asian: HR, 1.56; 95\% CI, 1.35-1.80, Figure S5A; Caucasian: HR, $1.37 ; 95 \%$ CI, 1.23-1.52, Figure S5B).

\section{Publication bias}

The publication bias of the included studies was assessed by funnel plots in this meta-analysis. Deeks' tests of publication bias in diagnostic analysis showed that there was no publication bias ( $p=0.634)$. Similarly, the publication bias was assessed for the association of CEA and OS in pancreatic cancer patients. Begg's tests showed that publication bias was not significant for the enrolled studies (Begg's test: $p=0.213)$. The funnel plot is shown in Figure S6.

\section{Discussion}

Traditional surgical specimens or biopsy tissues are used in the diagnosis of pancreatic cancer and considered the gold standard for clinical examination. However, limitations still exist, because they involve invasive procedures and delayed reflection of tumor dynamic changes. ${ }^{24,25}$ Hence, the current

Table 2 Characteristics of the diagnosis part of included studies

\begin{tabular}{|c|c|c|c|c|c|}
\hline Study & Region & $\begin{array}{l}\text { Number of } \\
\text { participants }\end{array}$ & $\begin{array}{l}\text { CA19-9: } \\
\text { TP/FP/FN/TN }\end{array}$ & $\begin{array}{l}\text { CEA: } \\
\text { TP/FP/FN/TN }\end{array}$ & $\begin{array}{l}\text { CEA-based tumor panel: TP/FP/FN/TN } \\
\text { (panel composition) }\end{array}$ \\
\hline Benini et $\mathrm{a}^{36}$ & America & 193 & $|4 / 25 /||/| 43$ & $10 / 26 / 15 / 142$ & 23/39/2/129 (CEA, CAI9-9) \\
\hline Carpelan-Holmstrom et al ${ }^{39}$ & Finland & 191 & $24 / 31 / 6 / 130$ & $9 / 7 / 21 / 154$ & - \\
\hline Haglund ${ }^{32}$ & Finland & 201 & $74 / 24 / 21 / 82$ & $47 / 25 / 40 / 81$ & $31 / 1 / 56 / 105$ (CEA, CAI25, CA19-9) \\
\hline Sakamoto et al ${ }^{38}$ & Japan & 61 & $26 / 2 / 4 / 29$ & $20 / 7 / 10 / 24$ & 28/9/2/22 (CEA, CAI9-9) \\
\hline Ni et $\mathrm{a}^{33}$ & China & 205 & $84 / 57 / 21 / 43$ & $47 / 25 / 58 / 75$ & 39/16/66/84 (CEA, CAI9-9) \\
\hline Del Favero et $\mathrm{al}^{34}$ & Italy & 139 & 20/33/9/77 & $8 / 24 / 21 / 86$ & 6/5/23/105 (CEA, CAI9-9) \\
\hline Liao et $\mathrm{al}^{35}$ & China & 150 & $84 / 15 / 28 / 23$ & $37 / 2 / 75 / 36$ & 40/I/72/37 (CEA, CA19-9, CA50, CA242) \\
\hline Duraker et $\mathrm{al}^{37}$ & Turkey & 181 & $100 / 14 / 23 / 44$ & $48 / 5 / 75 / 53$ & 42/3/8I/55 (CEA, CAI9-9) \\
\hline Gu et $\mathrm{al}^{31}$ & China & 132 & $43 / 33 / 9 / 47$ & $28 / 35 / 24 / 45$ & 47/5/5/75 (CEA, CAI9-9, CAI25, CA242) \\
\hline
\end{tabular}

Note: -, indicates not mentioned.

Abbreviations: CA19-9, carbohydrate antigen 19-9; CEA, carcinoembryonic antigen; FN, false negative; FP, false positive; TN, true negative; TP, true positive. 


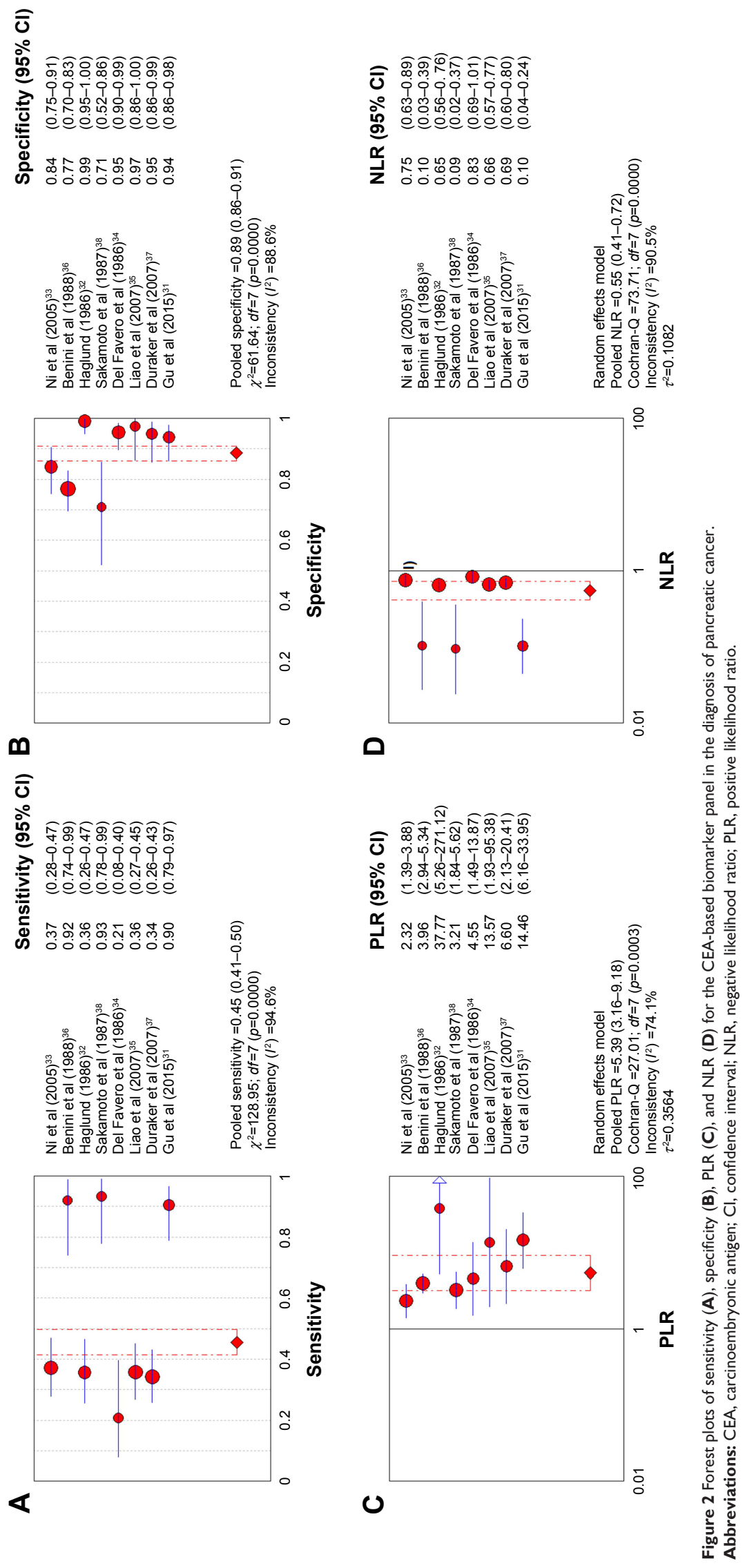


Table 3 Pooled diagnostic accuracy

\begin{tabular}{|c|c|c|c|c|c|c|c|}
\hline $\begin{array}{l}\text { Study } \\
\text { participants }\end{array}$ & $\begin{array}{l}\text { Diagnostic } \\
\text { biomarker }\end{array}$ & Sensitivity $(95 \% \mathrm{Cl})$ & Specificity $(95 \% \mathrm{Cl})$ & PLR (95\% Cl) & NLR $(95 \% \mathrm{Cl})$ & DOR (95\% Cl) & $\begin{array}{l}\text { AUC } \\
\text { (SEM) }\end{array}$ \\
\hline \multirow[t]{3}{*}{$P C$ vs non-PC } & CAI9-9 & $0.78(0.75-0.8 \mathrm{I})$ & $0.73(0.69-0.76)$ & $2.77(1.98-3.87)$ & $0.34(0.27-0.43)$ & $8.44(5.12-\mid 3.91)$ & 0.81 \\
\hline & CEA & $0.43(0.39-0.47)$ & $0.82(0.79-0.84)$ & $2.40(1.68-3.43)$ & 0.7 I $(0.65-0.78)$ & $3.57(2.29-5.57)$ & 0.61 \\
\hline & CEA-based panel & $0.45(0.4 I-0.50)$ & $0.89(0.86-0.91)$ & $5.39(3.16-9.18)$ & $0.55(0.4 I-0.72)$ & $19.20(6.45-57.18)$ & 0.90 \\
\hline Heterogeneity, & CA19-9 & $28.1 \%(0.194)$ & $90.0 \%(0.000)$ & $85.9 \%(0.000)$ & $46.2 \%(0.06 I)$ & $68.4 \%(0.001)$ & - \\
\hline \multirow[t]{2}{*}{$P^{2}(p$-value $)$} & CEA & $67.1 \%(0.002)$ & $89.0 \%(0.000)$ & $69.2 \%(0.001)$ & $26.8 \%(0.205)$ & $56.8 \%(0.017)$ & - \\
\hline & CEA-based panel & $94.6 \%(0.000)$ & $88.6 \%(0.000)$ & $74.1 \%(0.000)$ & $90.5 \%(0.000)$ & $81.7 \%(0.000)$ & - \\
\hline
\end{tabular}

Note: -, indicates not mentioned.

Abbreviations: AUC, area under the curve; CA19-9, carbohydrate antigen 19-9; CEA, carcinoembryonic antigen; CI, confidence interval; DOR, diagnostic odds ratio; NLR, negative likelihood ratio; PC, pancreatic cancer; PLR, positive likelihood ratio; SEM, standard error of the mean.

attempts to develop screening tests for early diagnosis have predominantly focused on serum biomarkers. The application of CA19-9, a widespread screening tool for pancreatic cancer, has been restricted due to its low sensitivity and specificity. CA19-9 is elevated in patients with other upper gastrointestinal tumors, biliary obstruction, and other benign conditions. ${ }^{26}$ Furthermore, Lewis antigen-negative individuals, constituting approximately $5 \%-10 \%$ of the population, are genetically unable to produce CA19-9. ${ }^{27}$ Recently, combinations of biomarkers in a panel screen were shown to have increased power to accurately diagnose pancreatic cancer over any single marker alone. ${ }^{28-30}$ Serum CEA is the second most common biomarker used clinically for detecting pancreatic cancer. Many recent studies have shown that CEA, when combined with other biomarkers, including CA19-9, CA125, and CA50, may increase the accuracy in distinguishing cancer from normal patients. ${ }^{31-39}$
Moreover, many studies have reported that CEA could also play an important role in predicting survival of pancreatic cancer patients. ${ }^{13,31,40-48}$ However, the relationship between CEA and pancreatic cancer still remains unclear. Therefore, it is necessary to conduct a comprehensive analysis to assess the clinical utility of CEA in pancreatic cancer patient diagnosis and prognostic prediction.

In this meta-analysis, evidence showed that CEA-based panels were superior to CEA or CA19-9 alone in terms of test specificity, although the sensitivity of the panels had no obvious advantage. As an evaluation index of the overall performance of diagnostic tests, the pooled DOR of CEA-based panels was 19.20, indicating a significantly higher diagnostic accuracy compared with CEA or CA19-9 alone. Similarly, CEA-based panels had a better diagnostic capability (AUC $=0.90$ ) to indicate the risk of pancreatic cancer according to the suggested guidelines for the interpretation of the area under summary

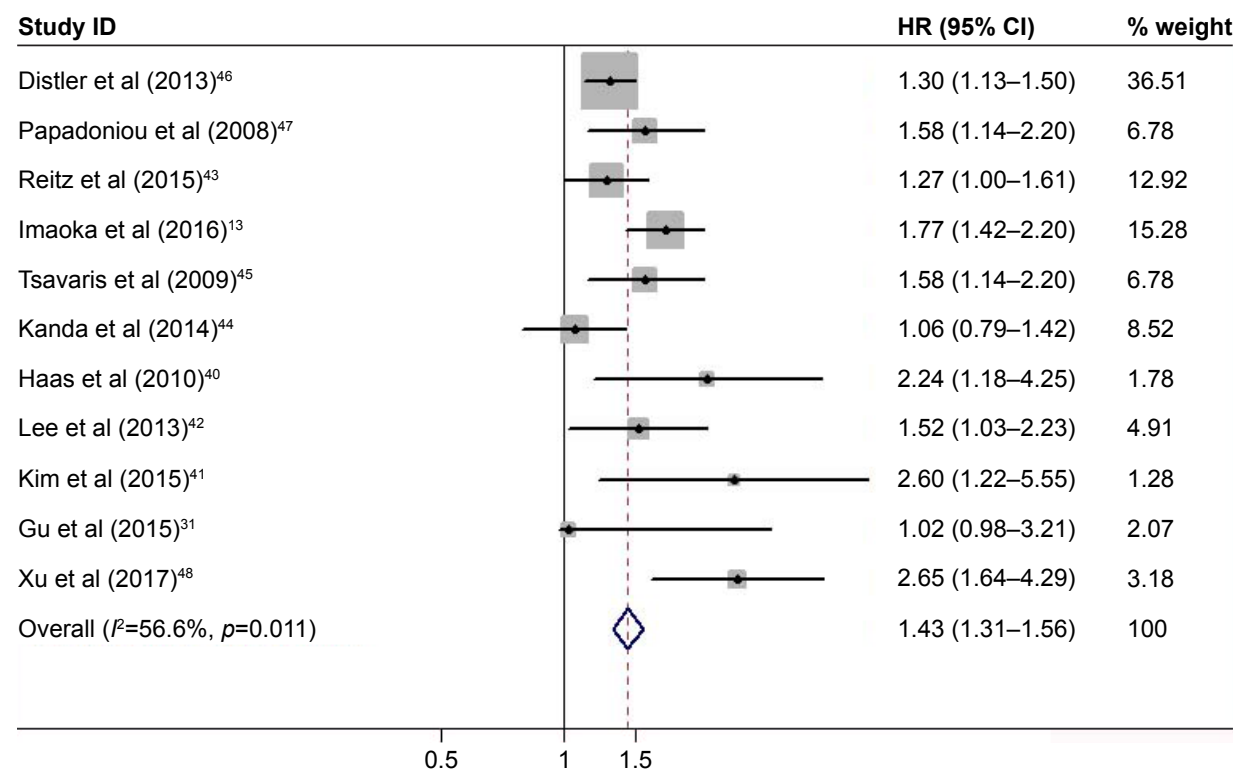

Figure 3 Forest plot of the HRs for survival with high serum CEA levels in pancreatic cancer. Abbreviations: CEA, carcinoembryonic antigen; $\mathrm{Cl}$, confidence interval; $\mathrm{HR}$, hazard ratio. 
receiver operating characteristic curve value. ${ }^{49}$ Hence, these results demonstrate the promising clinical value of the CEA-based diagnostic panel as a diagnostic biomarker.

With regard to prognostic value, the expression of CEA was significantly associated with OS of pancreatic cancer patients. Papadoniou et $\mathrm{a}^{47}$ found that increased levels of the markers CEA and CA19-9, which indicate a higher tumor burden, were also associated with a worse prognosis. Reitz et $\mathrm{a}^{43}$ reported that a linear combination of CEA and CA19-9 is significantly better for prognostic prediction compared with single tumor markers. Imaoka et $\mathrm{al}^{13}$ showed that CEA level is an independent prognostic factor in patients with metastatic pancreatic cancer, and a combination chemotherapy regimen (such as Folfirinox and nab-paclitaxel plus gemcitabine) may offer only a modest survival benefit in cases with high CEA. In this field, our team has published two relevant studies. ${ }^{14,48} \mathrm{We}$ previously showed that increases in serum CEA in combination with CA19-9 could significantly enhance the prognostic value of CA19-9. ${ }^{48} \mathrm{~A} \mathrm{CEA}^{+} / \mathrm{CA}_{125} / \mathrm{CA} 19-9 \geq 1,000 \mathrm{U} / \mathrm{mL}$ serum signature could better identify a subgroup of patients with poor response to radical resection. ${ }^{14}$

Several limitations in this meta-analysis study should be addressed. First, a relatively small number of studies with a limited number of subjects were included in this meta-analysis, which may reduce the statistical power for determining the diagnostic role of CEA for pancreatic cancer. Second, through subgroup analysis of ethnicity, we found that the differences in patient characteristics (age, country, and other variables) and cutoff criteria of the included studies may be another potential source of heterogeneity. Third, our study selected English language-only publications or unpublished articles, which may result in certain publication bias. Finally, the findings of this meta-analysis need to be confirmed by further multicenter, prospective clinical studies to enable a definitive conclusion to be made.

\section{Conclusion}

The current meta-analysis showed that a CEA-based panel is better at diagnosing pancreatic cancer than CA125 or CA19-9 alone. Furthermore, high levels of serum CEA are significantly related to poor prognosis of patients with pancreatic cancer. Thus, the measurement of serum CEA, as a vital supplementary to CA19-9, is inexpensive, convenient, and necessary for monitoring this disease.

\section{Acknowledgments}

This review was jointly funded by the National Science Fund for Distinguished Young Scholars of China (No 81625016), and Shanghai Sailing Program (17YF1402500). Qingcai Meng, Si Shi and Chen Liang shared the first authorship.

\section{Disclosure}

The authors report no conflicts of interest in this work.

\section{References}

1. Siegel RL, Miller KD, Jemal A. Cancer Statistics, 2017. CA Cancer J Clin. 2017;67(1):7-30.

2. Hidalgo M. Pancreatic cancer. N Engl J Med. 2010;362(17): 1605-1617.

3. Petrushnko W, Gundara JS, De Reuver PR, O'Grady G, Samra JS, Mittal A. Systematic review of peri-operative prognostic biomarkers in pancreatic ductal adenocarcinoma. HPB (Oxford). 2016;18(8): 652-663.

4. Gold P, Freedman SO. Specific carcinoembryonic antigens of the human digestive system. $J$ Exp Med. 1965;122(3):467-481.

5. Molina R, Barak V, van Dalen A, et al. Tumor markers in breast cancerEuropean Group on Tumor Markers recommendations. Tumour Biol. 2005;26(6):281-293.

6. Grunnet M, Sorensen JB. Carcinoembryonic antigen (CEA) as tumor marker in lung cancer. Lung Cancer. 2012;76(2):138-143.

7. Juweid M, Sharkey RM, Behr T, et al. Improved detection of medullary thyroid cancer with radiolabeled antibodies to carcinoembryonic antigen. J Clin Oncol. 1996;14(4):1209-1217.

8. Nazli O, Bozdag AD, Tansug T, Kir R, Kaymak E. The diagnostic importance of CEA and CA 19-9 for the early diagnosis of pancreatic carcinoma. Hepatogastroenterology. 2000;47(36):1750-1752.

9. Satake K, Chung YS, Yokomatsu H, et al. A clinical evaluation of various tumor markers for the diagnosis of pancreatic cancer. Int $J$ Pancreatol. 1990; 7(1-3):25-36.

10. Hata S, Sakamoto Y, Yamamoto Y, et al. Prognostic impact of postoperative serum CA 19-9 levels in patients with resectable pancreatic cancer. Ann Surg Oncol. 2012;19(2):636-641.

11. Hamanaka Y, Hamanaka S, Suzuki M. Sialyl Lewis(a) ganglioside in pancreatic cancer tissue correlates with the serum CA 19-9 level. Pancreas. 1996;13(2):160-165.

12. Takasaki H, Uchida E, Tempero MA, Burnett DA, Metzgar RS, Pour PM. Correlative study on expression of CA 19-9 and DU-PAN-2 in tumor tissue and in serum of pancreatic cancer patients. Cancer Res. 1988; 48(6):1435-1438.

13. Imaoka H, Mizuno N, Hara K, et al. Prognostic impact of carcinoembryonic antigen (CEA) on patients with metastatic pancreatic cancer: a retrospective cohort study. Pancreatology. 2016;16(5):859-864.

14. Liu L, Xu H, Wang W, et al. A preoperative serum signature of CEA+/CA125+/CA19-9 $\geq 1000 \mathrm{U} / \mathrm{mL}$ indicates poor outcome to pancreatectomy for pancreatic cancer. Int J Cancer. 2015;136(9): 2216-2227.

15. Moher D, Liberati A, Tetzlaff J, Altman DG, Group P. Preferred reporting items for systematic reviews and meta-analyses: the PRISMA statement. Int J Surg. 2010;8(5):336-341.

16. Whiting PF, Rutjes AW, Westwood ME, et al. QUADAS-2: a revised tool for the quality assessment of diagnostic accuracy studies. Ann Intern Med. 2011;155(8):529-536.

17. Margulis AV, Pladevall M, Riera-Guardia N, et al. Quality assessment of observational studies in a drug-safety systematic review, comparison of two tools: the Newcastle-Ottawa Scale and the RTI item bank. Clin Epidemiol. 2014;6:359-368.

18. Hayden JA, Cote P, Bombardier C. Evaluation of the quality of prognosis studies in systematic reviews. Ann Intern Med. 2006;144(6): 427-437.

19. Tierney JF, Stewart LA, Ghersi D, Burdett S, Sydes MR. Practical methods for incorporating summary time-to-event data into meta-analysis. Trials. 2007;8:16. 
20. Parmar MK, Torri V, Stewart L. Extracting summary statistics to perform meta-analyses of the published literature for survival endpoints. Stat Med. 1998;17(24):2815-2834.

21. Walter SD. Properties of the summary receiver operating characteristic (SROC) curve for diagnostic test data. Stat Med. 2002;21(9): $1237-1256$

22. DerSimonian R, Laird N. Meta-analysis in clinical trials revisited. Contemp Clin Trials. 2015;45(pt A):139-145.

23. Rosman AS, Korsten MA. Application of summary receiver operating characteristics (sROC) analysis to diagnostic clinical testing. Adv Med Sci. 2007;52:76-82.

24. Rosch T, Dittler HJ, Strobel K, et al. Endoscopic ultrasound criteria for vascular invasion in the staging of cancer of the head of the pancreas: a blind reevaluation of videotapes. Gastrointest Endosc. 2000;52(4): 469-477.

25. Vargas R, Nino-Murcia M, Trueblood W, Jeffrey RB Jr. MDCT in Pancreatic adenocarcinoma: prediction of vascular invasion and resectability using a multiphasic technique with curved planar reformations. AJR Am J Roentgenol. 2004;182(2):419-425.

26. Locker GY, Hamilton S, Harris J, et al. ASCO 2006 update of recommendations for the use of tumor markers in gastrointestinal cancer. J Clin Oncol. 2006;24(33):5313-5327.

27. Tempero MA, Uchida E, Takasaki H, Burnett DA, Steplewski Z, Pour PM. Relationship of carbohydrate antigen 19-9 and Lewis antigens in pancreatic cancer. Cancer Res. 1987;47(20):5501-5503.

28. Koopmann J, Fedarko NS, Jain A, et al. Evaluation of osteopontin as biomarker for pancreatic adenocarcinoma. Cancer Epidemiol Biomarkers Prev. 2004;13(3):487-491.

29. Kuhlmann KF, van Till JW, Boermeester MA, et al. Evaluation of matrix metalloproteinase 7 in plasma and pancreatic juice as a biomarker for pancreatic cancer. Cancer Epidemiol Biomarkers Prev. 2007; 16(5):886-891.

30. Simeone DM, Ji B, Banerjee M, et al. CEACAM1, a novel serum biomarker for pancreatic cancer. Pancreas. 2007;34(4):436-443.

31. Gu YL, Lan C, Pei H, Yang SN, Liu YF, Xiao LL. Applicative value of serum CA19-9, CEA, CA125 and CA242 in diagnosis and prognosis for patients with pancreatic cancer treated by concurrent chemoradiotherapy. Asian Pac J Cancer Prev. 2015;16(15):6569-6573.

32. Haglund $C$. Tumour marker antigen CA125 in pancreatic cancer: a comparison with CA19-9 and CEA. Br J Cancer. 1986;54(6):897-901.

33. Ni XG, Bai XF, Mao YL, et al. The clinical value of serum CEA, CA19-9, and CA242 in the diagnosis and prognosis of pancreatic cancer. Eur J Surg Oncol. 2005;31(2):164-169.

34. Del Favero G, Fabris C, Plebani M, et al. CA 19-9 and carcinoembryonic antigen in pancreatic cancer diagnosis. Cancer. 1986;57(8): 1576-1579.

35. Liao Q, Zhao YP, Yang YC, Li LJ, Long X, Han SM. Combined detection of serum tumor markers for differential diagnosis of solid lesions located at the pancreatic head. Hepatobiliary Pancreat Dis Int. 2007;6(6):641-645.

36. Benini L, Cavallini G, Zordan D, et al. A clinical evaluation of monoclonal (CA19-9, CA50, CA12-5) and polyclonal (CEA, TPA) antibodydefined antigens for the diagnosis of pancreatic cancer. Pancreas. 1988;3(1):61-66.
37. Duraker N, Hot S, Polat Y, Hobek A, Gencler N, Urhan NCEA. CA 19-9, and CA 125 in the differential diagnosis of benign and malignant pancreatic diseases with or without jaundice. J Surg Oncol. 2007; 95(2):142-147.

38. Sakamoto K, Haga Y, Yoshimura R, Egami H, Yokoyama Y, Akagi M. Comparative effectiveness of the tumour diagnostics, CA 19-9, CA 125 and carcinoembryonic antigen in patients with diseases of the digestive system. Gut. 1987;28(3):323-329.

39. Carpelan-Holmstrom M, Louhimo J, Stenman UH, Alfthan H, Haglund C. CEA, CA 19-9 and CA 72-4 improve the diagnostic accuracy in gastrointestinal cancers. Anticancer Res. 2002;22(4):2311-2316.

40. Haas M, Laubender RP, Stieber P, et al. Prognostic relevance of CA 19-9, CEA, CRP, and LDH kinetics in patients treated with palliative second-line therapy for advanced pancreatic cancer. Tumour Biol. 2010;31(4):351-357.

41. Kim J, Lee YS, Hwang IK, et al. Postoperative carcinoembryonic antigen as a complementary tumor marker of carbohydrate antigen 19-9 in pancreatic ductal adenocarcinoma. J Korean Med Sci. 2015; 30(3):259-263.

42. Lee KJ, Yi SW, Chung MJ, et al. Serum CA 19-9 and CEA levels as a prognostic factor in pancreatic adenocarcinoma. Yonsei Med J. 2013; 54(3):643-649.

43. Reitz D, Gerger A, Seidel J, et al. Combination of tumour markers CEA and CA19-9 improves the prognostic prediction in patients with pancreatic cancer. J Clin Pathol. 2015;68(6):427-433.

44. Kanda M, Fujii T, Takami H, et al. Combination of the serum carbohydrate antigen 19-9 and carcinoembryonic antigen is a simple and accurate predictor of mortality in pancreatic cancer patients. Surg Today. 2014;44(9):1692-1701.

45. Tsavaris N, Kosmas C, Papadoniou N, et al. CEA and CA-19.9 serum tumor markers as prognostic factors in patients with locally advanced (unresectable) or metastatic pancreatic adenocarcinoma: a retrospective analysis. J Chemother. 2009;21(6):673-680.

46. Distler M, Pilarsky E, Kersting S, Grutzmann R. Preoperative CEA and CA 19-9 are prognostic markers for survival after curative resection for ductal adenocarcinoma of the pancreas - a retrospective tumor marker prognostic study. Int J Surg. 2013;11(10):1067-1072.

47. Papadoniou N, Kosmas C, Gennatas K, et al. Prognostic factors in patients with locally advanced (unresectable) or metastatic pancreatic adenocarcinoma: a retrospective analysis. Anticancer Res. 2008; 28(1B):543-549.

48. Xu HX, Liu L, Xiang JF, et al. Postoperative serum CEA and CA125 levels are supplementary to perioperative CA19-9 levels in predicting operative outcomes of pancreatic ductal adenocarcinoma. Surgery. 2017;161(2):373-384.

49. Swets JA. Measuring the accuracy of diagnostic systems. Science. 1988;240(4857):1285-1293.
OncoTargets and Therapy

\section{Publish your work in this journal}

OncoTargets and Therapy is an international, peer-reviewed, open access journal focusing on the pathological basis of all cancers, potential targets for therapy and treatment protocols employed to improve the management of cancer patients. The journal also focuses on the impact of management programs and new therapeutic agents and protocols on
Dovepress

patient perspectives such as quality of life, adherence and satisfaction The manuscript management system is completely online and includes a very quick and fair peer-review system, which is all easy to use. Visit http://www.dovepress.com/testimonials.php to read real quotes from published authors. 